\section{Coarse-Grained Picture for Controlling Complex Quantum Systems}

\author{
Toshiya TAKAMI ${ }^{1 *}$ and Hiroshi FuJISAKI ${ }^{2}$ \\ ${ }^{1}$ Institute for Molecular Science, Myodaiji, Okazaki, \\ 444-8585, Japan \\ 2 Department of Chemistry, Boston University, 590 \\ Commonwealth Ave., Boston, Massachusetts, 02215, USA
}

KEYWORDS: random matrix, quantum chaos, coarse-grain, optimal control, rotating-wave approximation

Controlling atomic and molecular processes by laser fields is one of current topics in physics and chemistry, and there are various control schemes applied to such processes. ${ }^{1}$ These strategies are known to work when the system to be controlled is rather simple or small. However, in reality, the system can become "complex," where the dynamics will be described by multi-level-multi-level transitions with a random interaction. Although the laser field can be obtained by optimal control theory $(\mathrm{OCT})^{1}$ even for such complex systems, it is difficult to analyze the controlled dynamics because such a field obtained numerically is often too complicated to interpret. Hence it is desirable to have a more analytical point of view.

It is well known that the $\pi$ pulse $^{2}$ or its generalizations can be employed to control few-level problems. ${ }^{3}$ Recently, an analytic result for multi-level control problems between general quantum states has been reported. ${ }^{4}$ The scheme is based on STIRAP, ${ }^{1}$ and assumes an intermediate state coupled to the initial and target states. Though the scheme can accomplish perfect control, it relies on the energy level picture of a quantum system, so it is difficult to apply the scheme to large systems.

In this short note, we propose a new approach to obtain an analytic optimal field for complex quantum systems. ${ }^{5}$ Under a "coarse-grained picture" with OCT, which is valid for such a complex system, we derive an analytic expression for the optimal field which steers initial states to target states in a certain limit. By numerically solving Schrödinger equations, we confirm that perfect control is actually achieved. This point is important because the zeroth-order solutions of OCT, ${ }^{6,7}$ which look similar to our result, are not guaranteed to achieve perfect control. Another point is that our final expression does not require a detailed information from the energy level picture, so that it is easy to apply to large quantum systems, in principle.

We use OCT as a theoretical vehicle. The aim of OCT is to obtain an optimal field $\epsilon(t)$ which guides the system from an initial state $\left|\Phi_{i}\right\rangle$ at $t=0$ to a target state $\left|\Phi_{f}\right\rangle$ at some specific time $t=T$. According to the OCT scheme by Zhu, Botina, and Rabitz, ${ }^{1,8}$ the optimal field for the Hamiltonian $H[\epsilon(t)]=H_{0}+\epsilon(t) V$ is given by

$$
\epsilon(t)=\frac{1}{\alpha \hbar} \operatorname{Im}[\langle\phi(t) \mid \chi(t)\rangle\langle\chi(t)|V| \phi(t)\rangle],
$$

\footnotetext{
*Present address: Computing and Communications Center, Kyushu University, Fukuoka, 812-8581, Japan.
}

where the quantum state $|\phi(t)\rangle$ and the inverselyevolving quantum state $|\chi(t)\rangle$ satisfy usual Schrödinger equations with boundary conditions $|\phi(0)\rangle=\left|\Phi_{i}\right\rangle$ and $|\chi(T)\rangle=\left|\Phi_{f}\right\rangle$. In order to maximize the final overlap $J_{0}=\left|\left\langle\phi(T) \mid \Phi_{f}\right\rangle\right|^{2}$, numerical iterations ${ }^{8}$ are necessary.

We approximately solve this problem by introducing "coarse-grained (CG)" Rabi states. We extend the usual Rabi oscillation between two eigenstates to the oscillation between two time-dependent states, $\left|\phi_{0}(t)\right\rangle$ and $\left|\chi_{0}(t)\right\rangle$, defined by

$$
\left|\phi_{0}(t)\right\rangle \equiv U_{0}(t, 0)\left|\Phi_{i}\right\rangle, \quad\left|\chi_{0}(t)\right\rangle \equiv U_{0}(t, T)\left|\Phi_{f}\right\rangle
$$

where $U_{0}\left(t_{2}, t_{1}\right)$ is the propagator for $\epsilon(t)=0$ from $t_{1}$ to $t_{2}$. We introduce the CG Rabi states as

$$
\begin{array}{cc}
|\phi(t)\rangle= & \left|\phi_{0}(t)\right\rangle \cos \Omega t-i e^{-i \theta}\left|\chi_{0}(t)\right\rangle \sin \Omega t, \\
|\chi(t)\rangle= & -i e^{i \theta}\left|\phi_{0}(t)\right\rangle \sin \Omega(t-T) \\
& +\left|\chi_{0}(t)\right\rangle \cos \Omega(t-T) .
\end{array}
$$

The phase $\theta$ is determined from a normalization condition $\langle\phi(t) \mid \phi(t)\rangle=1[$ or $\langle\chi(t) \mid \chi(t)\rangle=1]$ as

$$
e^{2 i \theta}=\frac{\left\langle\phi_{0}(t) \mid \chi_{0}(t)\right\rangle}{\left\langle\chi_{0}(t) \mid \phi_{0}(t)\right\rangle}=\frac{\left\langle\phi_{0}(T) \mid \Phi_{f}\right\rangle}{\left\langle\Phi_{f} \mid \phi_{0}(T)\right\rangle}
$$

and $\Omega$ is a constant determined later. It is also shown ${ }^{9}$ that the CG Rabi states (3) and (4) satisfy Schrödinger equations with the optimal field derived below when $\left|\Phi_{i}\right\rangle$ and $\left|\Phi_{f}\right\rangle$ contain many eigenstates without a special correlation, i.e., they are random vectors, ${ }^{5}$ and the target time $T$ is long enough.

Substituting eqs. (3) and (4) into eq. (1), and after some manipulations, we have an expression for the optimal field ${ }^{9,10}$

$$
\epsilon(t)=\frac{\sin 2 \Omega T}{2 \alpha \hbar} \operatorname{Re}\left[e^{-i \theta}\left\langle\phi_{0}(t)|V| \chi_{0}(t)\right\rangle\right] .
$$

We have used $\left|\left\langle\phi_{0}(t) \mid \chi_{0}(t)\right\rangle\right| \ll 1$ which is justified when $\left|\Phi_{i}\right\rangle$ and $\left|\Phi_{f}\right\rangle$ are random vectors as above.

Substituting eq. (3) [or (4)] into the Schrödinger equation with the field (6), and invoking the rotating-wave approximation (RWA), i.e., omitting highly oscillating terms ${ }^{2}$ we have

$$
\Omega=\frac{\bar{V}^{2} \sin 2 \Omega T}{4 \alpha \hbar^{2}}
$$

where

$$
\bar{V}=\sqrt{\lim _{\tau \rightarrow \infty} \frac{1}{\tau} \int_{0}^{\tau}\left|\left\langle\phi_{0}(t)|V| \chi_{0}(t)\right\rangle\right|^{2} d t}
$$

is an "average transition element" between the timedependent states (2). These equations constitute a set of equations to determine the value of $\Omega$ for given $\alpha$ and $T$. The final overlap follows as

$$
J_{0}=\sin ^{2} \Omega T,
$$

and the amplitude of the optimal field $\bar{\epsilon}$ is given by

$$
\bar{\epsilon} \equiv \sqrt{\lim _{\tau \rightarrow \infty} \frac{1}{\tau} \int_{0}^{\tau}|\epsilon(t)|^{2} d t}=\frac{\sqrt{2} \hbar \Omega}{\bar{V}} .
$$

Furthermore we set perfect control $J_{0}=1$, which is realized when $\Omega=\pi / 2 T$ [See eq. (9)]. This is equivalent 


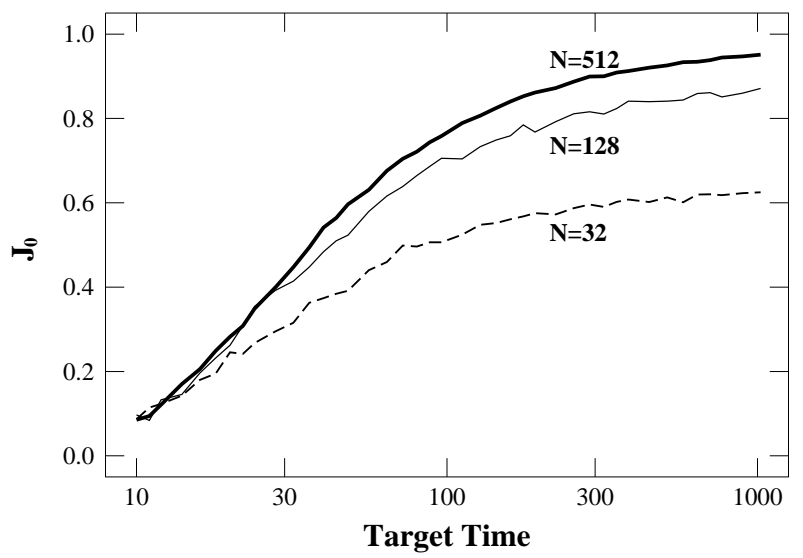

Fig. 1. The final overlap driven by the analytic optimal field, eq. (11), is shown as a function of the target time $T$. The system is random matrices with size $N$. The initial and target states are random vectors with Gaussian random elements, and the average $J_{0}$ 's for 100 ensembles are depicted.
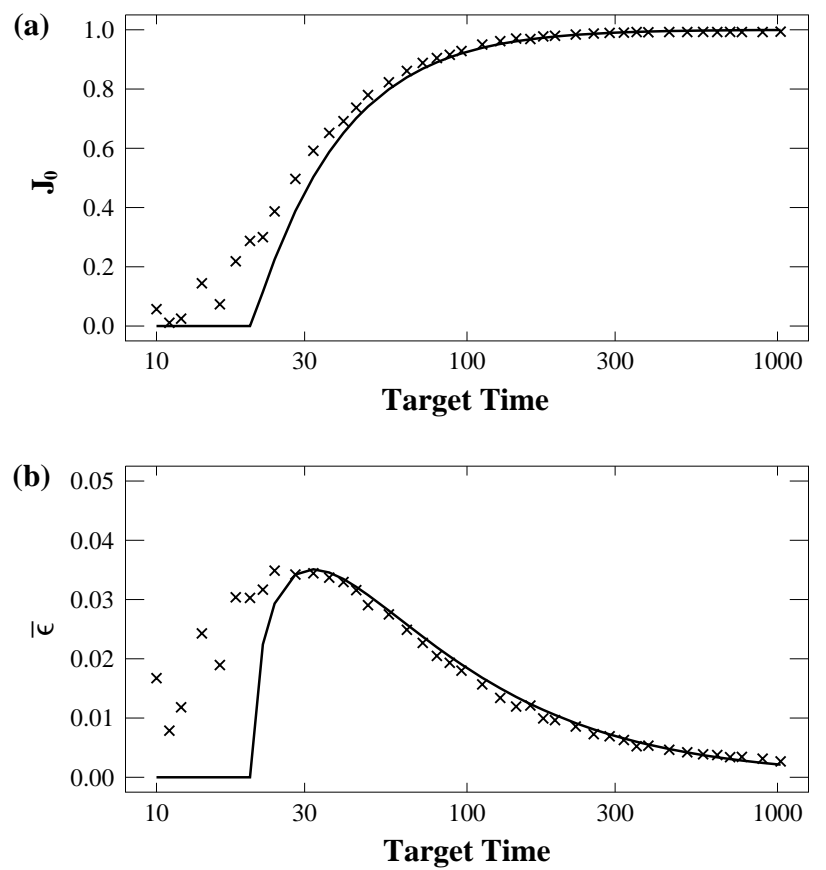

Fig. 2. (a) The final overlap $J_{0}=\left|\left\langle\phi(T) \mid \Phi_{f}\right\rangle\right|^{2}$ and (b) the averaged field amplitude $\bar{\epsilon}$ are shown as a function of the target time $T$. Marks $(\times)$ represent numerical results by solving OCT equations using the Zhu-Botina-Rabitz scheme. ${ }^{8}$ Solid curves represent our analytic results using the CG Rabi state.

to take the limit $\alpha \rightarrow 0$ [See eq. (7)]. Substituting eq. (7) into eq. (6), and using $\Omega=\pi / 2 T$, we obtain the final expression,

$$
\epsilon(t)=\frac{\pi \hbar}{\bar{V}^{2} T} \operatorname{Re}\left[e^{-i \theta}\left\langle\phi_{0}(t)|V| \chi_{0}(t)\right\rangle\right] .
$$

Note that this contains the same factor $\left\langle\phi_{0}(t)|V| \chi_{0}(t)\right\rangle$ as the zeroth-order solution of OCT, ${ }^{6,7}$ but the prefactor is introduced for perfect control. Actually the pulse area $A(t)$ can be defined by

$$
A(t) \equiv\left|\int_{0}^{t} \epsilon\left(t^{\prime}\right) \frac{2\left\langle\phi_{0}\left(t^{\prime}\right)|V| \chi_{0}\left(t^{\prime}\right)\right\rangle}{\hbar} d t^{\prime}\right|,
$$

as an extension of the usual two-state case. ${ }^{2}$ If we substitute eq. (11) into eq. (12), and use eq. (8), we obtain $A(T)=\pi$ under RWA, i.e., eq. (11) is a generalized $\pi$ pulse.

In Fig. 1, we show that the analytic field (11) actually works $\left(J_{0} \rightarrow 1\right)$ for $N \times N$ random matrix systems. ${ }^{5}$ Since we have used the RWA and omitted $\mathcal{O}(1 / \sqrt{N})$ terms in the derivation of eq. (11), perfect control is achieved when $T, N \rightarrow \infty$ as expected. In Figs. 2(a) and 2(b), we confirm eqs. (9) and (10) by comparing with numerical calculations using the Zhu-Botina-Rabitz scheme. ${ }^{8}$ The agreement is good, especially for large $T$.

We have studied random matrix systems as "complex" quantum systems, while real systems are located between simple systems and random matrix systems. Hence it will be interesting to apply this analytic field to more realistic cases of banded random matrix systems, i.e., a transition dipole matrix $V$ is a sparse matrix and there can be no direct transitions between arbitrary quantum states. Our analytic field often fails to control in the situation that single-photon processes are forbidden, while we can show that nearly perfect control is achieved ${ }^{10}$ if we restrict the initial and target state. Even in such a case, the next order solution is easily constructed along our picture, ${ }^{10}$ and can be applicable to multi-photon processes.

The authors thank Prof. S.A. Rice, Prof. H. Rabitz, and Prof. M. Toda for useful discussions. The research of the authors has been supported in part by JSPS Grantin-Aid No. 14077213.

1) S. A. Rice and M. Zhao, Optical Control of Molecular Dynamics (John Wiley \& Sons, New York, 2000); M. Shapiro and P. Brumer, Principles of the Quantum Control of Molecular Processes (John Wiley \& Sons, New York, 2003).

2) L. Allen and J. H. Eberly, Optical Resonance and Two-level Atoms (Dover, New York, 1987).

3) Y. Teranishi and H. Nakamura: Phys. Rev. Lett. 81 (1998) 2032; K. Nagaya, Y. Teranishi and H. Nakamura: J. Chem. Phys. 117 (2002) 9588; H. Fujisaki, Y. Teranishi and H. Nakamura: J. Theor. Comp. Chem. 1 (2002) 245.

4) P. Král, Z. Amitay and M. Shapiro: Phys. Rev. Lett. 89 (2002) 063002.

5) F. Haake: Quantum Signatures of Chaos, 2nd ed. (SpringerVerlag, Heidelberg, 2001).

6) V. Dubov and H. Rabitz: Chem. Phys. Lett. 235 (1995) 309; J. Chem. Phys. 103 (1995) 8412.

7) W. Zhu and H. Rabitz: J. Chem. Phys. 110 (1999) 7142.

8) W. Zhu, J. Botina and H. Rabitz: J. Chem. Phys. 108 (1998) 1953.

9) T. Takami, H. Fujisaki and T. Miyadera: Adv. Chem. Phys. (in press); e-print nlin.CD/0402005.

10) T. Takami and H. Fujisaki: unpublished. 